\title{
A Proposed Neuro-Fuzzy Model For Adult Asthma Disease Diagnosis
}

\author{
Somdatta Patra ${ }^{1}$ and Mr. GourSundarMitra Thakur ${ }^{2}$ \\ ${ }^{1}$ Department of Computer Science and Engineering, \\ Lovely Professional University, Punjab, India \\ Somdattapatra156@gmail.com \\ ${ }^{2}$ Assistant professor of the Department of Computer Science and Engineering, \\ Lovely Professional University, Punjab, India \\ cse.gsmt@gmail.com
}

\begin{abstract}
The task of medical diagnosis with the help different intelligent system techniques is always crucial because it require high level of accuracy and less time consumption in decision making. Among all other AI techniques Artificial Neural Networks (ANN) as a tool for medical diagnosis has become the most popular in last few decades due to its flexibility and accuracy. ANN was developed after getting the inspiration from biological neurons. There are various diseases that are still needed to be diagnosed. Among many other critical diseases like cancer, thyroid disorder, diabetes, heart diseases, neuro diseases, asthma disease was also tried to bediagnosed effectively with various ANN mechanisms by different researchers. Due to various uncertainties about symptoms the study of Neuro-Fuzzy technique in this context became very popular in last few years. Neuro-Fuzzy now-a-days is one of the most advanced technique that is mainly concatenation of two model-neural networks and the fuzzy logic. In this model various parameters are used that are much crucial if ill-chosen and may led to failure of the whole system. Recent trend in analysis is following this model for advanced expert work. In this study an enhanced Neuro-fuzzy model has been proposed for the proper diagnosis of adult Asthma disease and to foster the proper aid or medication to the patients and make physicians alert for the upcoming disease pattern otherwise they may lack in the process of providing improper medication at right time. In the first phase data collected from various hospitals are used to train by three different types of learning of ANN like ANN with Self Organizing Maps (SOM), ANN with Learning Vector Quantization (LVQ) and ANN with Backpropagation Algorithm (BPA) through NF tool for much accurate result. In the second phase fuzzy rule base is applied to the classified data for the diagnosis of the disease.
\end{abstract}

\section{KEYWORDS}

Adult asthma, Artificial Neural Network (ANN), Neuro Fuzzy (NF), Learning Vector Quantization (LVQ), Self-Organizing Map (SOM),Backpropagation.

\section{INTRODUCTION}

The physician with information and advance knowledge may sometime not able to make proper or justifiable judgements in the medical field. Only the clinical data and empirical studies is not enough to make optimal decisions. Sometimes clinical data may skip the needed information at right time at in right place. So the need of computer assisted decision support system is needed to intelligently filter the right disease and to foster the better medication to the needy patients

Rupak Bhattacharyya et al. (Eds) : ACER 2013, 
Asthma is one of the chronic inflammatory diseases now prevailing world wide and affecting huge mass of the population including the youths and the adults. It has been seen that from very starting of the information system, the health care system is one of the important field that has ever found. This medical service is mainly used for the medication of the human being. As it was also told "Prevention is better than cure". So pre-diagnosis is very much important for the prevention. Mass of the population is suffering from many diseases like eye diseases, cardiac diseases, cancers, tumors, pulmonary diseases, brain cancers and tumors, urinary diseases, etc. These all diseases are much chronic to diagnose and it is hard to take preventive measures for those.

'Medical diagnosis' word is actually stating its meaning that diagnosis in medical field. It is done to identify a probable disease and also to know how to cure that one. During diagnosis tests are needed to be done as per the reference of the doctors or the advisors. There are various diseases and their categorization can be done as infectious diseases, contagious diseases, communicable diseases, non-communicable diseases, etc.These diseases are needed to be properly diagnosed.

Only the simple medical methods are not enough to easily diagnose a disease and effectively. So with the advancement of the modern technology, we need to do something new and eye catchy. Side by side of various medical and scientific methods there are so much computational methods that was already been started in the year 1970 with the development of MYCIN at Stanford University.

MYCIN was actually a type of expert systems that had used the computer applications so as to embody some of the non-algorithmic expertise that could solve certain types of problems. For example, expert systems can be used for diagnostic purposes that service both for people and machinery. They also can perform decisions making, monitoring real time systems, do work as like human expertise. Earliest expert system MYCIN was developed so as to identify the bacteria that might cause severe diseases and could be resolved by the implementation of the specific antibiotics in the body.

Cancer is a malignant disease that causes unregulated and uncontrolled growth of the cells in the body. Earlier the cancer diagnosis was done by the methods of Laser- induced Fluorescence (LIF) and Raman Spectra and nanoparticles (breast cancer) that combine laser spectroscopy, biomedical, nanoparticle removing technology with specific target agents and computer technology [1,2]. Again Incremental Background Knowledge has been used in medical field for diagnosing the breast cancer for introducing the unlabeled data into proper training sets [3]. But with the advancement of expert system an evolutionary type of NF model [4] has been developed and also by using the fuzzy rules the cost effective models [5] has also been developed for breast cancer diagnosis and its treatment.

Thyroid disease is one of the hormonal disorders that causes either hyperthyroidism (Grave's disease, pituitary gland malfunctioning) or hypothyroidism (intake of excessive iodine). This disease can be diagnosed by clinical evaluation, blood tests (T3, T4), imaging tests, biopsies, and other tests like saliva and urine testing. Ultrasound elastography for FNA biopsy for thyroid diagnosis was developed earlier [6]. But it takes long time to perform the tests medically and scientifically. This conventional method has now been replaced by the application of expert system for prognosis of this thyroid disease [7]. Again Progressive Learning Vector Quantization Neural Network (PLVQNN) is used for thyroid disease diagnosis with thyroid segmentation and volume estimation [8].

Similarly asthma disease is needed to be diagnosed. It is one of the chronic diseases that can be either diagnosed by applying the scientific methods or the expertise methods. 
In the below sections the short description about the Asthma disease is given. Then in the nest subsection there is brief note on Adult Asthma and its prevalence. After that in the nest proceeding sections the knowledge of expert system and NF has been provided for medical diagnosis. After that the methodology of my proposed work has been described thoroughly. Lastly conclusion and references for the proposed work has been made.

\section{ASTHMA}

Asthma is one of the common occurring chronic inflammatory diseases where actually effect found on the airways that are mainly used for respiration .i.e. inhale of oxygen and exhale of carbon di-oxide.It may occur at any age. The inner walls of an asthmatic's airways actually get swollen creating difficulty in breathing. Here appears the change that is either gets stimulated by the allergens or by the environmental triggers. Airway inflammation plays a significant role among wheezing, asthma, cystic fibrosis (CF) and chronic obstructive pulmonary disease (COPD). So there is a need to identify the airway inflammatory changes.

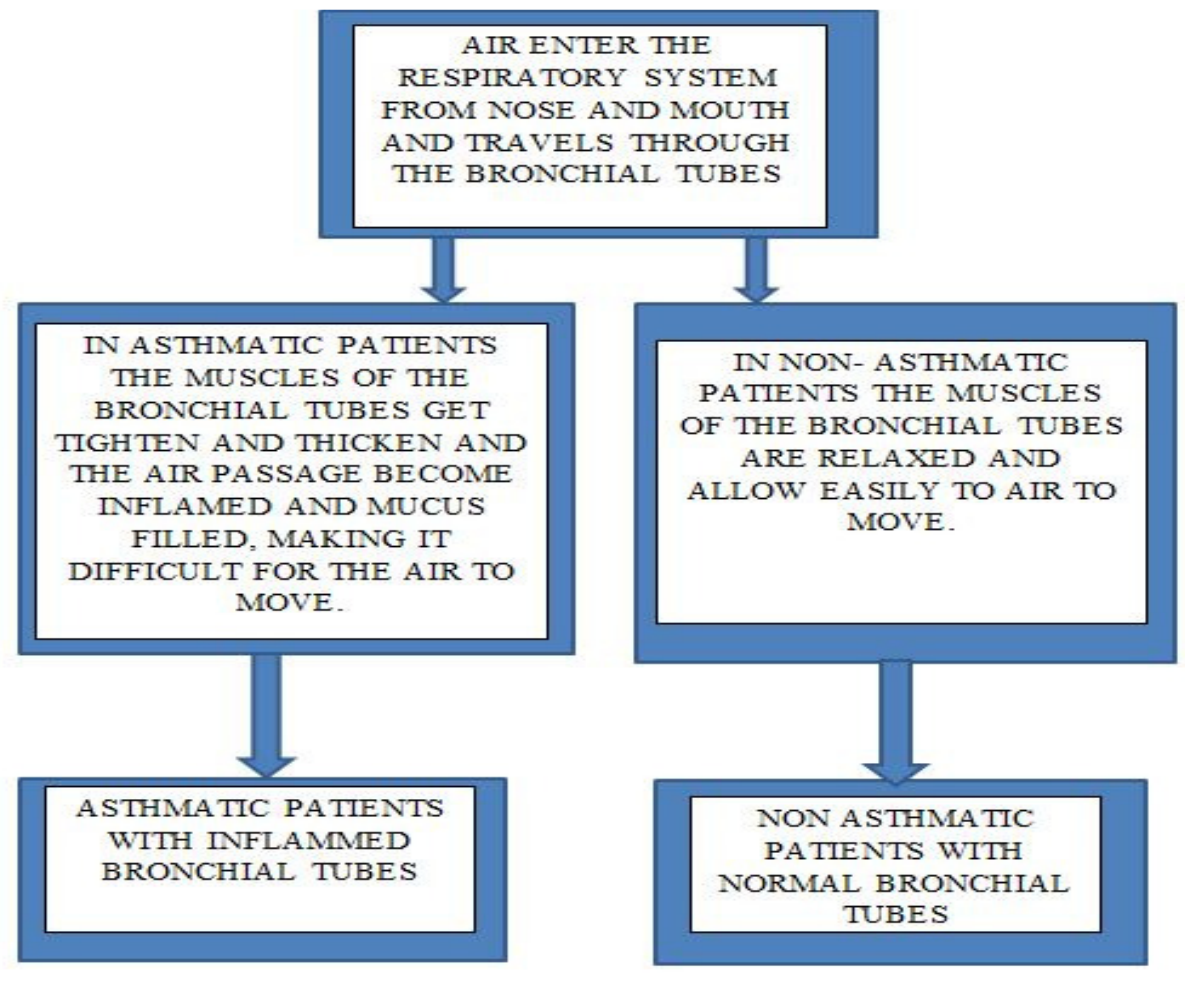

Figure 1. Asthma affecting human lungs

Symptoms of asthma are wheezing, dysponea, coughing, fever, nausea, loss of appetite, etc. Changes that appear in Asthma disease give response like:

- Hyper-reactive Response

- Inflammatory Response

Asthma disease is found globally but specially affecting the peoples of developing countries. The term 'poorly controlled asthma',we meant to say that the increase and instant rate of morbidity, mortality and socio-economic are found. Asthma management is needed to be the essential plan for asthma control. 
It is one of the prevailing diseases in our country India. It can also be caused by the addition of environmental as well as genetic factors.

As per the survey it has been noted that estimation of every one in 15 persons is suffering from this type of lung or pulmonary disease. It is a type of chronic disorder that notaffects only the adult but also the childhood. The prevalence and occurrence of this childhood asthma is increasing day-by-day. It is actually causing huge problems as like considerable morbidity, disability and occasional mortality at all ages. In between 12-15 million people in the United States near about 5 million children are having Asthma and for those patients about 3 million doctors are allotted the work of visiting to those patients every hour.

Asthma is one of the incurable illnesses. Although good treatment and management available but still people cannot live normally healthy.

\subsection{Adult Asthma}

When asthma is diagnosed at an age older than 20 is called as adult-onset asthma. It is estimated that about half of adults have asthma with allergies.

Who may have adult-onset asthma are as follows [9]:

- Women having hormonal changes, who are pregnant or who are experiencing menopause.

- Women take estrogen in a regular manner due to menopause for 10 years or longer.

- People with certain cold or flu.

- People who have the pets in their home.

People do smoking in a regular manner or work in the place with much mold, dust or irritants. Asthma in adult human body is called as adult-asthma.

\subsection{Prevalence of Asthma}

There are mainly three metrics that are used to describe asthma prevalence [9]. These are as follow:

- Lifetime prevalence which is used to measure how many individuals in the population will be diagnosed with asthma for at least once in his or her lifetime.

- Current prevalence that is used to measure how many individuals will be diagnosed recently with asthma in a given year.

- Attack prevalence that is used to measure how many individuals in the population who are asthma attack for a given year? 
Table 1.Prevalence of Asthma.

\begin{tabular}{|c|c|}
\hline AGE /GENDER CATEGORY & $\begin{array}{c}\text { ASTHMA PREVALENCE RATE PER 1000 } \\
\text { POPULATION (YEAR 2001) }\end{array}$ \\
\hline GIRLS OF AGE 0-17 & 74.4 \\
\hline BOYS OF AGE 0-17 & 99.0 \\
\hline ALL FEMALES & 82.6 \\
\hline ALL MALES & 63.6 \\
\hline OVERALL FAMILY & 73.4 \\
\hline
\end{tabular}

From the above chart it has been observable that females are having 10 percent higher lifetime prevalence rate than that of the male population.

In 2001 the current prevalence rate of the U.S. population who actually had asthma was estimated about 73.4 per 1000 persons. The highest and the current prevalence that was found at the age of 5-17 years were of rate 98.1 per 1000 persons.

The latest prevalence rate in females of 82.6 in per head of 1000 persons was about of 30 percent more than that for male population of 63.6 per head of 1000 persons. But for the children the estimated current asthma prevalence rate for boys was approximately of 30 percent more than for girl.

Earlier by using the scientific and medical methods the asthma can be diagnosed by the measuring the complexity of airflow pattern with the changes in the entropy by Approximate Entropy method (ApEnQ) [10]. Again diagnosis can be done by checking the frequency of the cough sound using wavelet analysis. These methods are long term. So to improve the process there is the emergence of the expert system [9].

\section{EXPERT SySTEM IN MEDICAL DiAgnOSIS}

Health care issues in the worldwide are increasing its prevalence in many countries by the upcoming trends of urbanization and also the industrialization that is mostly prevailing in many areas. In our country India alone has burden of the people suffering due to various diseases are more than 15 million patients. Despite the existence of effective medicines, many peoples are suffering due to the disease asthma. By observing the earlier studies it has been found that the important factor that leads to under-treatment of asthma are due to the non-availability and also the under-usage of the basic tools as like the spirometer for diagnosis and also for managing the asthma. Further, there are some other reasons like the non-availability of essential inhalation medicines mostly in the public sector hospitals and also the low affordability by the people of the developing countries make asthma management difficult.

It has been noted that the poor knowledge of both patients and doctors cause barrier in the effective treatment and also the management of asthma. So there is a need for some expert method that can do some better diagnosis in the field of computer science as well as in the medical field.

Expert system can be efficiently applied in the field of medicine. This system is more flexible as compared to that of the conventional system as it is not so much improved one and here testing of each and every patient are done every time and then medicines are prescribed. So it is a long task to do for every-one. So the emergence of the expert system has come into its way. 


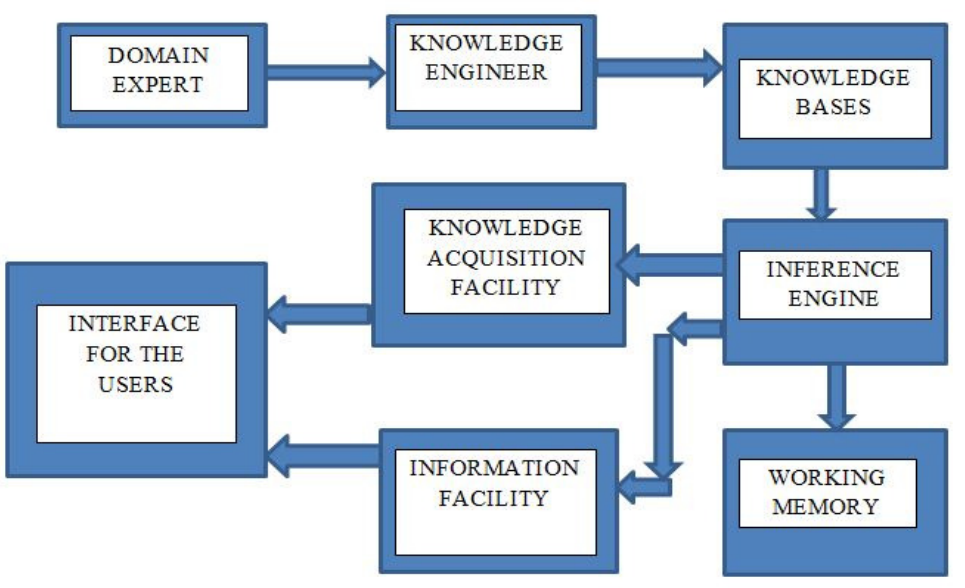

Figure 2. Expert system

These are the components of the expert system that are needed to be considered when we are going for making any system that works with expertize methods.

Various works has been done on various diseases like Thyroid, Cancer, Diabetes, etc. for Diabetes disease identification and proper medical an automated system was developed that eradicated the problem of communication gap between the patients and the doctors [18]. An expert system was developed for Cardiac disease diagnosis on the basis of various computing techniques as like fuzzy rule base learning, Genetic algorithm and ANN [19]. A fuzzy rule based system was developed for the diagnosis of liver disorder [20].Again an expert system was developed on the basis of supervised learning methodology for lung cancer diagnosis [22].

\section{NEURO-FuZZY SySTEM ARCHITECTURE}

Neuro fuzzy systems are a kind of enhancement over simple fuzzy expert systems. The term neuro-fuzzy derives from the terms ANN and fuzzy logic. This term neuro-fuzzy was proposed firstly by J. S. R. Jang. A NF system is actually based on a fuzzy system with a trained learning algorithm that has been developed from neural network. The learning procedure which is heuristic in nature mainly applies on the host data, and doing modification for making developed training sets with fuzzy system.

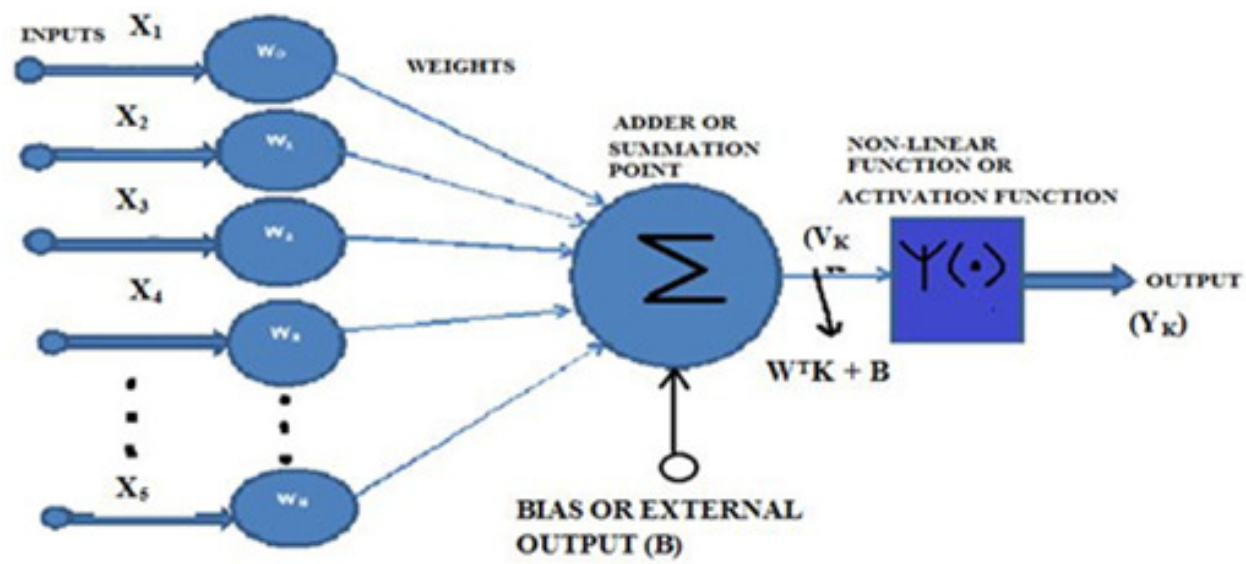

Figure 3. Artificial Neural Network (ANN) 


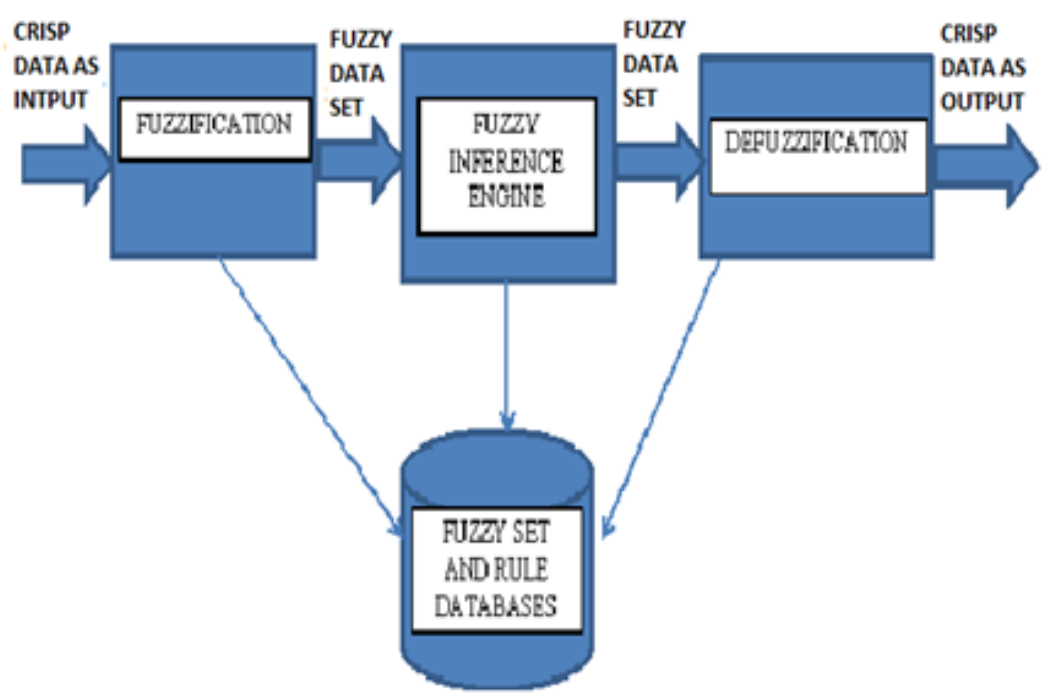

Figure 4. Neuro-Fuzzy (NF)

Neuro-fuzzy system is the aggregation of two techniques- one is the reasoning style of fuzzy systems and the other is the learning methodology of neural network. Neuro-fuzzy hybridization is widely termed as Fuzzy Neural Network (FNN). NF system uses reasoning style of fuzzy systems through the use of fuzzy sets that uses the concepts of the rule making with IF-THEN that is termed as the fuzzy rules.

- Neuro-fuzzy systems consist mainly of two requirements in fuzzy modeling that is contradictory in nature: interpretability versus accuracy. The model that describes the neuro-fuzzy technique with linguistic method is defined with the Mamdani model [20] and the model with accuracy is referred as the Takagi-Sugeno-Kang (TSK) model.

- The implementation of Neuro-Fuzzy Logic helps in probing the various approximation techniques from neural networks to get the exact parameter that are useful in the functioning of a fuzzy system.

Various works has already been done in diseases like diabetes, Thyroid, Cancer, etc. In Thyroid disease the work has already done on the basis of NF for diagnosing the disease with the symptoms and settings parameters for those symptoms so as to proper diagnosing the disease [7]. Similarly in Diabetes the work on fuzzy rule base system has done for diagnosing the disease with developing the decision support system for knowledge construction [15].Similarly in cancer disease various works has already been done. In Cancers like Ovarian, Oral and Cervical there has been a paper that describes the classification rules for chromatogram analysis by comparing the PCA and fuzzy clustering techniques with development of rules for diagnosis [16]. Similarly for identifying the sleeping stages in the child a neuro fuzzy classifier model was proposed that took inputs as the parameters of sleeping [17].

Fuzzy Rule-Base Expert System was developed for the evaluation of Asthma [11]. After developing the system the performance of the rules based system was checked. Again a decision support system was developed with five inputs and evaluating one output by designing the fuzzy inference system with fuzzy logics [12].

Only the expert system is not enough. So we have to move into the idea of more advanced technology that is neural network. 
Classification of Impulse Oscillometry (IOS) patterns are analyzed for lung functioning in asthma patients by artificial neural network [13].

Previous works are done on the medical diagnosis of asthma. But there is also need to develop a more improved model.The combination of the fuzzy technique with that of the expert system is helpful in developing an effective intelligent system that is required for evaluating the asthma expert's so as to derive a cost-effective intelligence systems for diagnosing the occurring of asthma disease in the adult human being [14].

In this study various symptoms for adult asthma are considered. Then an optimum NF model has been proposed where the performance of the models have been tested on 300 patients data collected by the sincere co-operation of Dr. Kanai LalPatra and Prof.J.L.Ghosh From Amri Super Speciality Hospital, Salt Lake, Kolkata- 700 028, Health Point Respiratory Care Clinic, Tarakeswar, Hooghly District, West Bengal-712410 And Tarakeswar Rural General Government Hospital, District: - Hooghly and R.G.Kar Medical College\& Hospital, Kolkata, West Bengal.

Here by using the various methods of learning like SOM, LVQ, BPA the proposed NF model has been compared. The accuracy of the systems istested by observing the results from the regression graph available in the NF tool of MATLAB and the best methods among them areproposed in my proposed work.

\section{NeUro FuZzy MOdel For ASTHMa DiseaSe}

The proposed paper is actually based firstly on neural network and various learning algorithm of neural network. As we know that there are various types of learning methods in neural network. This may be supervised, unsupervised or reinforcement learning's. By using the learning methods this paper further apply those in fuzzy environment so that development of the fuzzy sets done by creating the membership functions. After studying various papers on medical diagnosis for ASTHMA the method of learning are compared. By applying this, neuro-fuzzy techniques applied for diagnosing the disease bronchial ASTHMA on the basis of their symptoms named breathing problem, cough, and fever for days, nausea, wheezing, loss of appetite and dysponea. This diagnosis is done mainly for the patients"e medication and proper treatment. As ASTHMA is a chronic disorder, recurrent recovery measures to be taken.

In this paper diagnosis on Adult ASTHMA disease can be performed and we have to find best accuracy by comparing the performance by checking through the learning algorithms of back propagation. By using this we can get to know the best neuro-fuzzy network that give more effected result. Problem formulation is done based on the filtering out the real patients data, whose symptoms are much effective for disease diagnosis. The dataset for adult ASTHMA disease is obtained from medical reports of the patients that have been collected. In this paper approximately we are presenting 300 patients data with their symptoms and factors for checking and diagnosis of the disease.

The main aim or objective of the proposed work is to diagnosis the adult ASTHMA disease and applies algorithms of ANN with learning on the disease and also apply neuro-fuzzy classifier.

The very first step towards making the diagnosis for the disease is that we will collect the patients' data. The patients' data collection is done on the basis of their symptoms of the disease. Then we get theentirehealth condition history and other relevant facts. After that detailed medical examination with the relevant symptoms will be done. Patients present with or complain about certain symptoms. These are subjective reports. The physician or the doctors under that disease will carry out the medical tests so as to identify signs related to an illness. 
Proposed work follows the following steps:

1. Collection of the medical reports of the patients having Asthma or not having Asthma.

2. Making the dataset for the symptoms for Adult Asthma disease of the patients.

3. The Adult patients having asthma disease are specified with 1 and the patients not having Asthma are specified with 0 .

4. Feedback taken from doctors as well patients.

5. Perform classification of the patients using Backpropagation, LVQ, SOM learning methods and compare them to find the most accurate method.

6. Apply the rules of fuzzy.

7. Network will be developed that is chosen as a best network model for disease.

8. Provide patients with proper medication and treatment so that in future other will get alarm of the disease occurrence.

Research Methodology means the guideline that is used for solving a problem, with tasks, techniques and tools.

In this proposed work diagnosis on adult ASTHMA disease is performed on the basis of ANN learning algorithms. From the medical reports of patients, the data with same symptoms are taken and then making a diagnosis of those symptoms. After diagnosis the paper will show that how many patients are suffering from the disease and how many patients are not. The patients suffering from ASTHMA are coded 1 and others are coded as 0 . The learning algorithm will give more effected result that can formulate the hypothesis.

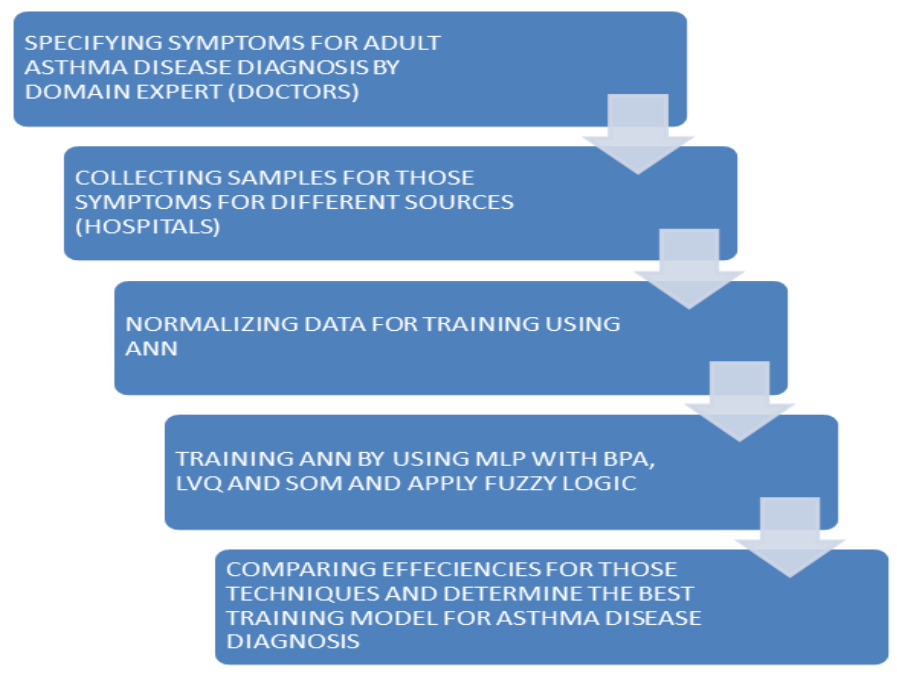

Figure 5. Research Methodology

\section{Design of The Proposed Model}

The medical diagnosis process of Asthmahappens with an individual patient consulting with the doctor. After consultation doctors set up a set of complaints named as symptoms of the disease. Data collection is done on the basis of patients past health condition, age (18 years and above), hygiene. A physical test is needed to be conducted, observations are analyzed and a medical treatment is needed to be started as per the reference of the doctors. Symptoms are arranged in specific orders and then they are made available for the classification. A treatment plan is needed 
to be proposed and physically the tests are performed to determine if the patient is having asthma disease. Neuro-fuzzy inference model is developed for Adult Asthma disease diagnosis.

The knowledge base has the entire set of the database. The fuzzy logic is set and neural networks provide the structure for the inference engine. The inference engine has the production rules that are fuzzy logic driven. The filters are present in the decision support engine for ranking the patients in terms of the presence or absence of Asthma. This representation is done in the computer system in the forms of binary where the presence of Asthma is represented by 1(input vector) and otherwise 0 (symptom vector).

The set of symptoms are poured into the network model so as to make the patients lists as per their symptoms of having the disease or not.

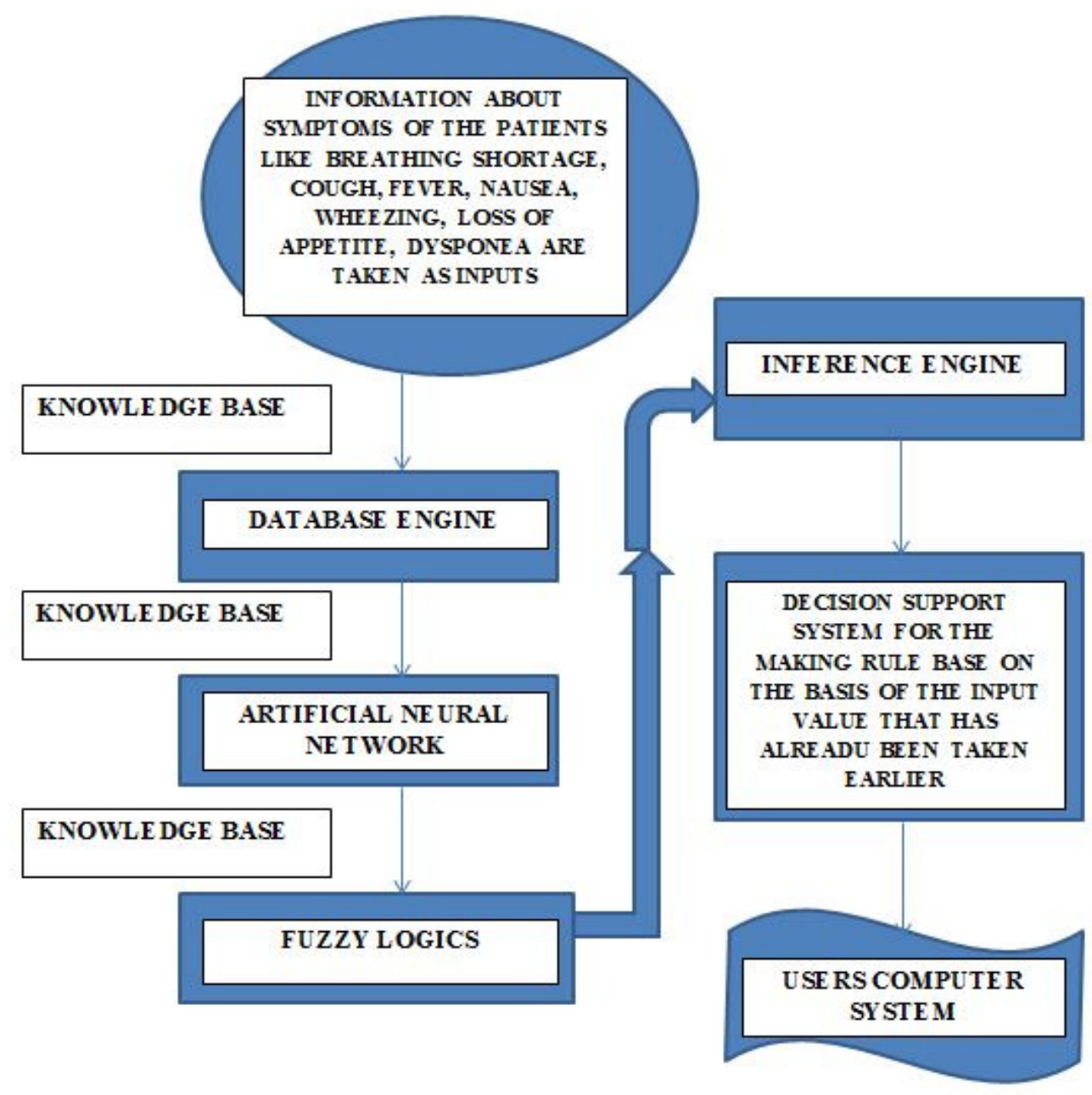

Figure 6. Neuro Fuzzy Model for Adult Asthma Diagnosis 


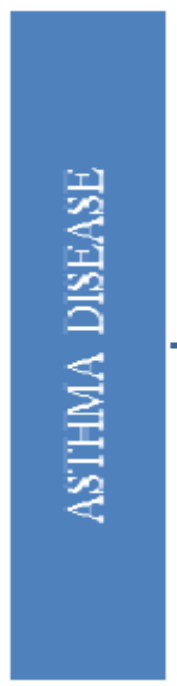

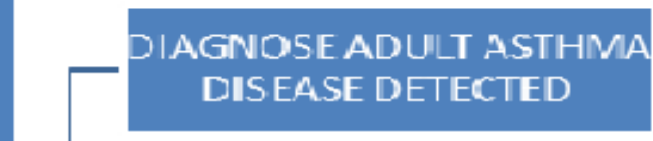

DIAGNOSE THATADULT

MIGHT HAVE ASTHMA

DISEASE

DIAGNOSE NO ADULT

ASTHMA DISEASE

DETECTED
WITH MORE THAN 5 SYMPTOMS

\author{
WITH ESTIMATELY 4 \\ SYMPTOMS \\ WITH 3 OR LESS THAN \\ 3 SYMPTOMS
}

Figure 7. Methodology to diagnose the Adult Asthma disease

\section{IMPLEMENTATION AND RESULT ANALYSIS}

In this section the implementation for the ASYHMA disease diagnosis has been shown by using the NF tool.

In the NF tool we have chosen about 300 patients data whom either having ASTHMA or not having ASTHMA. The patients having ASTHMA is coded by 1 otherwise awarded 0. Below snapshots are given after doing training of the data.

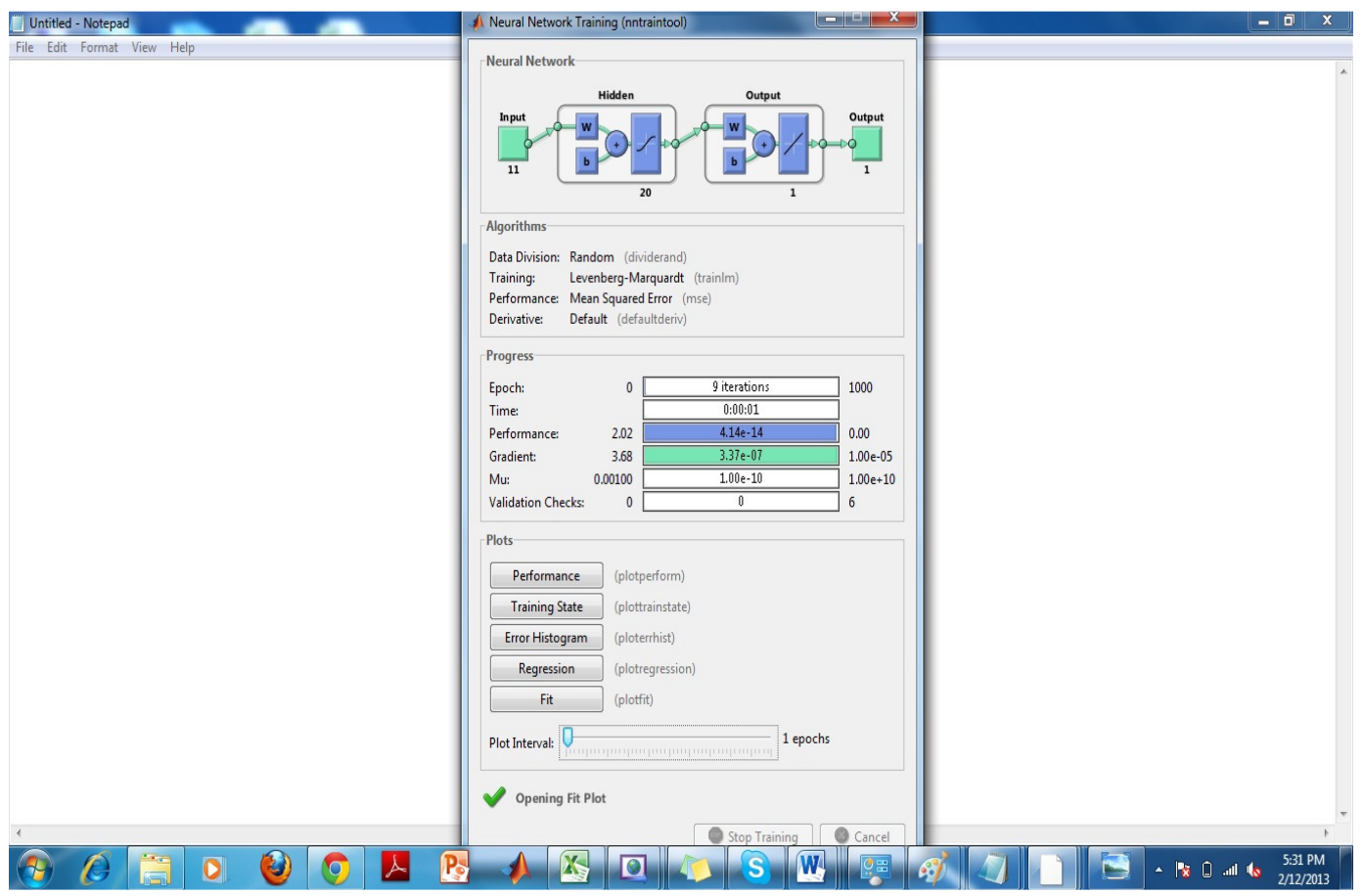

Figure 8. Analysis after training of the patients' data by NF tools 
In the above snapshot we have trained the data by using NF Fitting tool through Backpropagation Algorithm. Here Training of the data will get automatically stops when its generalization stops further improvement, as indicated by an increase in the mean square error of the validation samples. Out of 300, 210 samples are taken for training, 45 samples for testing and rest 45 are taken for validation. These all are done by calculating the Mean Squared Error value and regression value $R$. The mse value for 210 samples is $4.13641 \mathrm{e}-14$, for 45 testing samples is $3.13760 \mathrm{e}-14$ and finally for validation the mse value is of $5.35984 \mathrm{e}-14$.

Mean Squared Error (mse) is calculated as the average squared by considering the difference between outputs and targets. If we are having much lower values then our inputs consideration are much better. If we are getting Zero mean squared error then we are not getting any error value. This is actually taken for observing the performance of my system. From the above figure it has been observed that performance is 0 so no error found and also the snapshot proceeding to that performance is shown in below figure 9 .

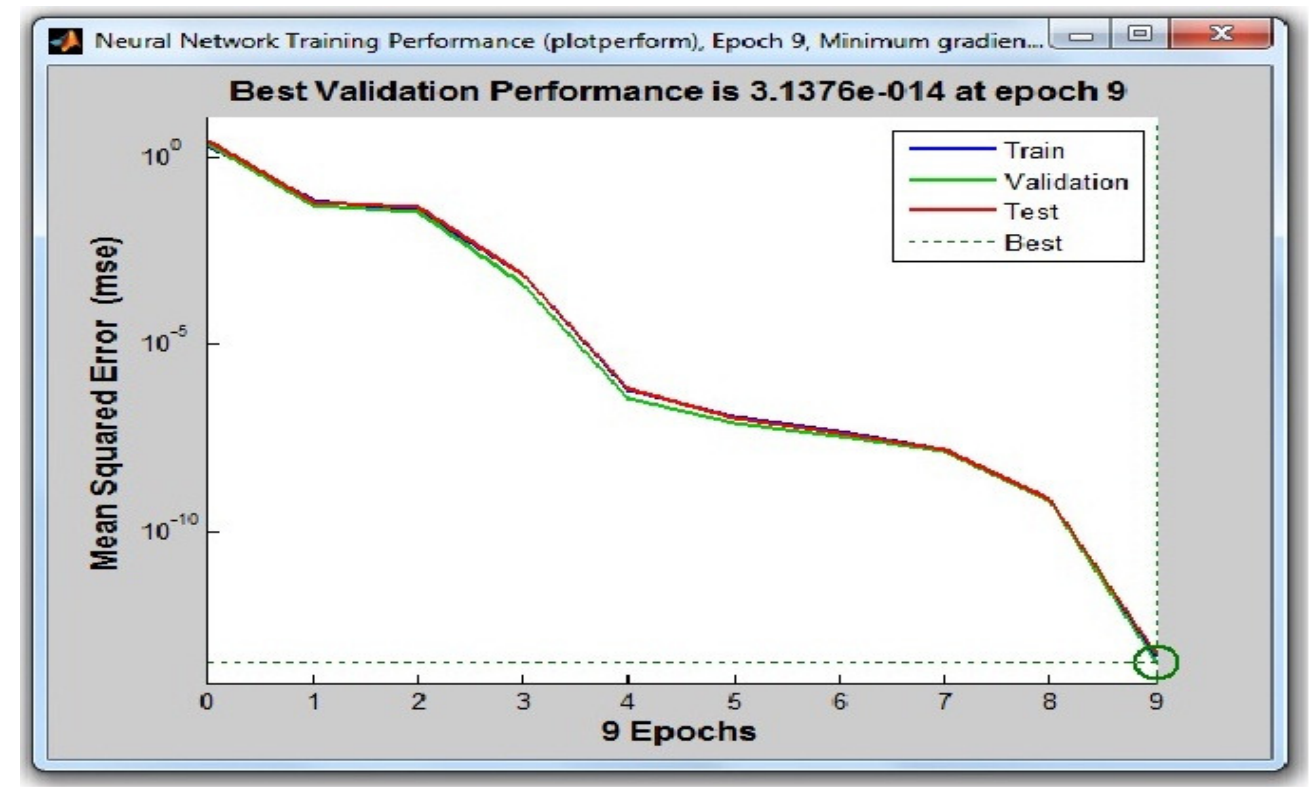

Figure 9. Performance validation with respect to mse and epochs

Here from the above snapshot it has been found that we are getting the best performance of about 3.1376e-014. This is found by 9 iterations called as epochs which are one of the steps in neural network process and its representation is iterations for 1000 epochs. It means the numbers of iterations are found for 1000 epochs and when we get the minimum iteration value for 1000 epochs then goal value for net.trainParam is achieved. So, there is a need to stop the training process.

Finally the error histogram is provided below for showing the accuracy of my proposed work. 


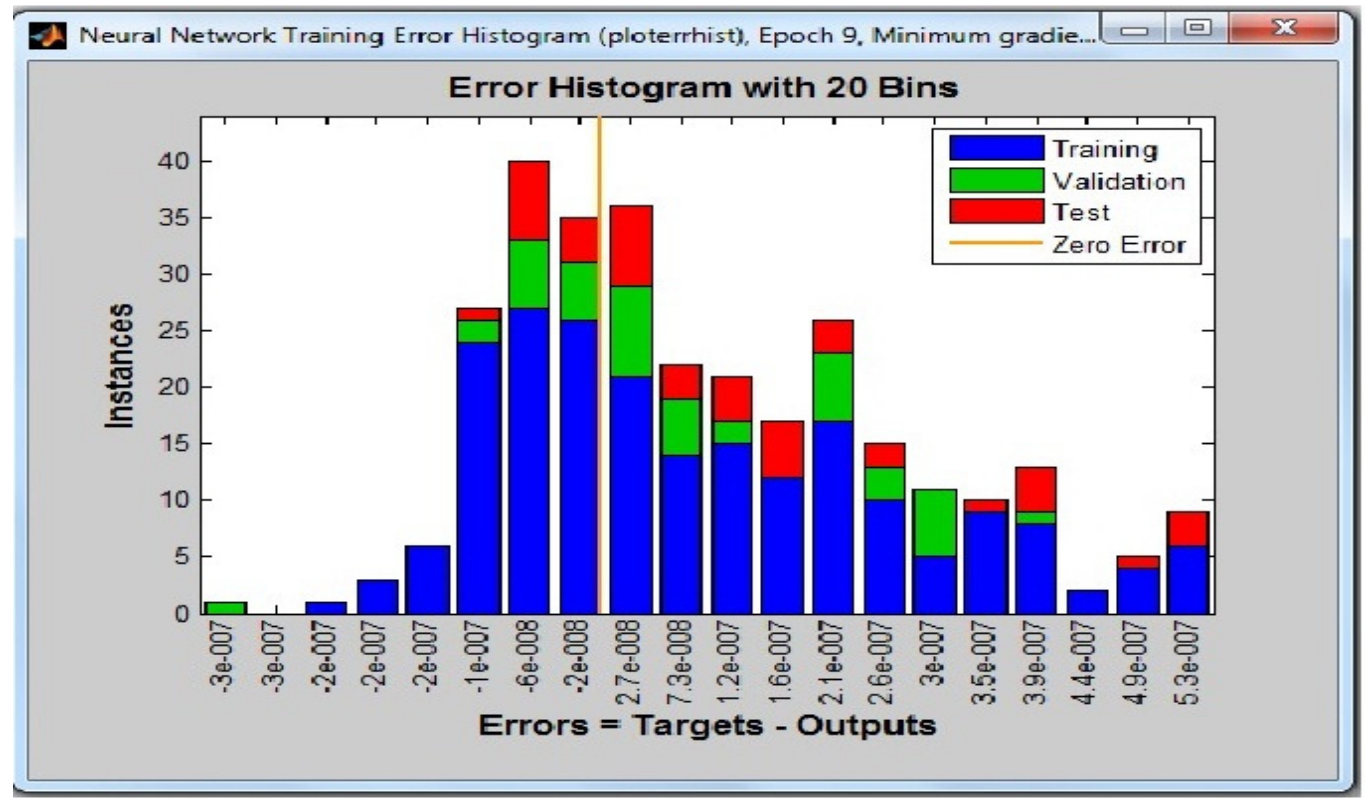

Figure 10. Error histogram diagram

\begin{tabular}{l} 
Neural Network Training Error Histogram (ploterrhist), Epoch 11, Minimum gradi... $\square$ \\
\hline
\end{tabular}

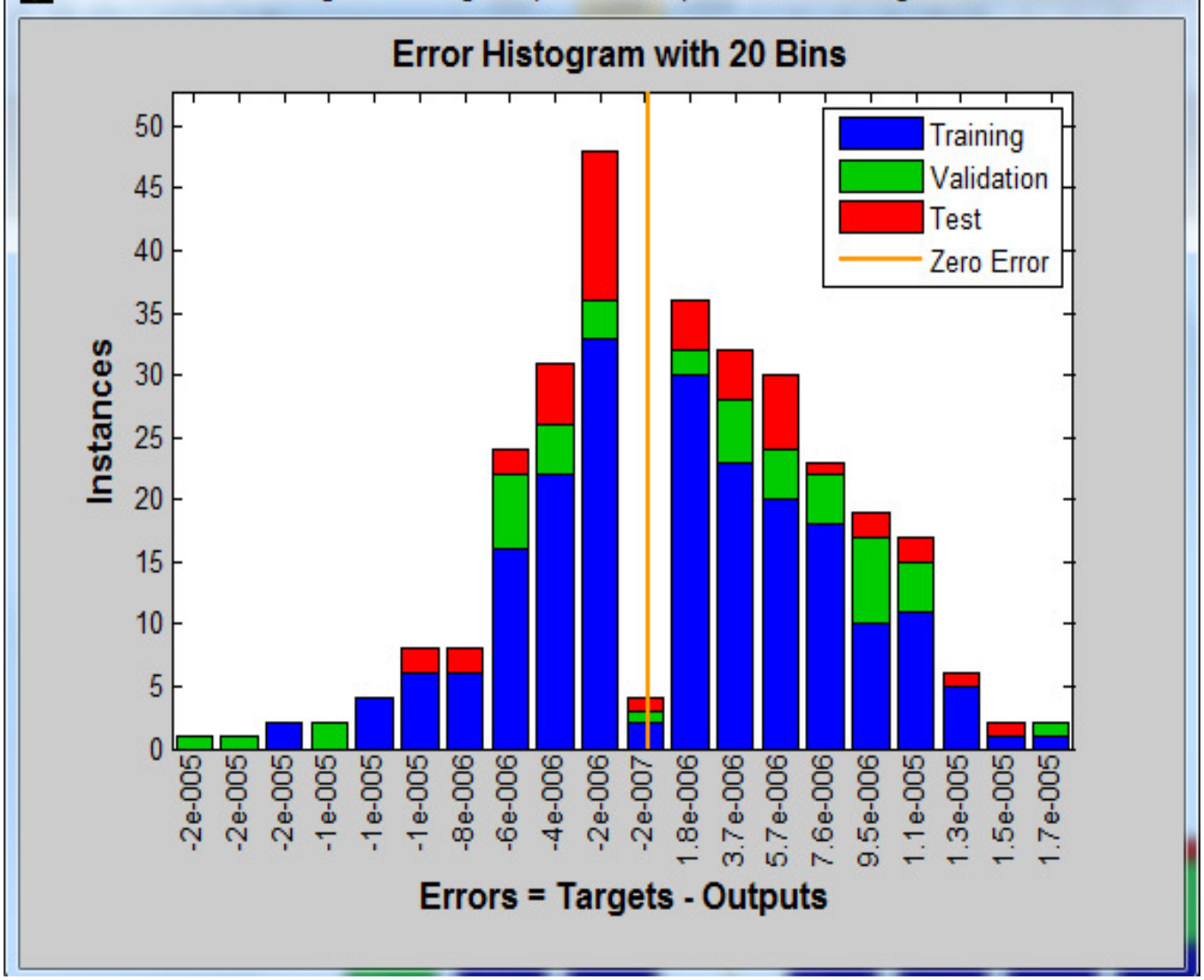

Figure 11. Error histogram after retraining 
From the above figure 10 it has been found that the error rate is much lower so giving much good result. If we get less error then we can say that our proposed work is running in the proper direction and can be done further with much better tool. After retraining is has been found the error rate is gradually decreasing after certain period of time and this is noted by noticing the value of mse.

\section{CONCLUSION}

Considering the prevalence of asthma and its increasing rate of the mortality and the morbidity, there is a need to develop an intelligence system that can diagnose adult asthma disease and can be applied to the hospitals for primary care with aims of:

1. Diagnosing.

2. Controlling with manageable measures.

3. Classifying the patients.

This main aim of this proposed work is actually to design asystem that is helpful for the diagnosis of adult Asthma using Neuro Fuzzy Logic. This will be helpful for the common people who suspects Asthma and will get the proper expected result. By observing those results proper and effective cure in diagnosing the Asthma are taken. The observed efficacy of the system developed is presented to the doctors so that they can give proper suggestion about the system to be developed in the field of observation. This system is available to all of the doctors through the internet facility. With consulting the doctors effective curative measures are to be taken as per their suggestion and also it is filled up in the proposed system to make available for further use. The efficiency of this system was analyzed using asthmatic patients data and acceptable result is developed. Future work can be done with more sensitive data for eradicating the system's good and effective performance. Tuning the neural network for better performance and applying the same model for other diseases. This will help in developing more developed system with less focus on the laboratory data

\section{REFERENCES}

[1] Xiaozhou Li, XingjiaGuo, Deli Wang, Yue wang, Xinyu Li, Ming Lei, Junxiu Lin, (2005), "Study of method and system for diagnosis of cancer using autofluorescence and Raman spectroscopy", Proceedings of the 2005 IEEE Engineering in Medicine and Biology 27th Annual Conference Shanghai, China, September 1-4, 2005, pp5453 - 5456.

[2] James Xing, Jie Zeng, Jing Yang1, Tao Kong, Tao Xu, Wilson Roa, Xiaoping Wang, and Jie Chen, (2007),"Gold-Based Nanoparticles for Breast Cancer Diagnosis and Treatment", publication IEEE 2007, pp2882 - 2885.

[3] Catarina Silva, Bernardete Ribeiro, Noel Lopes, (2010), "Improving recall values in breast cancer diagnosis with Incremental Background Knowledge", IEEE publication, pp1 - 6 .

[4] R. El Hamdi, M. Njah, M. Chtourou, (2010), "An evolutionary neuro-fuzzy approach to breast cancer diagnosis", IEEE publication, pp142-146.

[5] Gerald Schaefer and Tomoharu Nakashima , (2010), "Hybrid cost-sensitive fuzzy classification for breastcancer diagnosis", 32nd Annual International Conference of the IEEE EMBS Buenos Aires, Argentina, August 31 - September 4, 2010, pp6170-6173.

[6] Si Luo, Eung-Hun Kim, Manjiri Dighe and Yongmin Kim, (2009), "Screening of thyroid nodules by ultrasound elastography using diastolic strain variation ",31st Annual International Conference of the IEEE EMBS Minneapolis, Minnesota, USA, September 2-6, 2009, pp4420-4423.

[7] Imianvan Anthony Agboizebeta and Obi Jonathan Chukwuyeni, (2012), "Application of Neuro-Fuzzy Expert System for the Probe and Prognosis of Thyroid Disorder “, International Journal of Fuzzy Logic Systems (IJFLS) Vol.2, No.2, April 2012. 
[8] Chuan-Yu Chang and Yong-Cheng Hong Pau-Choo Chung, Chin-Hsiao Tseng, (2011), "A Neural Network for Thyroid Segmentation and Volume Estimation in CT Images “, IEEE publication 2011, pp43-55.

[9] Trends in Asthma Morbidity and Mortality. American Lung Association Epidemiology \& Statistics Unit. March 2003, https://aspe.hhs.gov/health/prevention/

[10] Juliana Veiga, Renan C. P. Faria, Guilherme P. Esteves, Agnaldo J. Lopes, José M. Jansen, and Pedro L. Melo, (2010), "Approximate entropy as a measure of the airflow pattern complexity in asthma ", 32nd Annual International Conference of the IEEE EMBS Buenos Aires, Argentina, August 31 September 4, 2010, pp2463-2466.

[11] M.H. Fazel Zarandi, M. Zolnoori, M. Moin and H. Heidarnejad, (2010), “”,Transaction E: Industrial Engineering Vol. 17, No. 2, pp129 -142.

[12] Ashish Patel Member IAENG, Jyotsna Choubey, Shailendra K Gupta, M. K. Verma Membe, IAENG, Rajendra Prasad, Qamar Rahman, (2012), "Decision Support System for the Diagnosis of Asthma Severity Using Fuzzy Logic", proceedings of International Multiconference of Engineers and Computer Scientists 2012, Vol I, IMECS, march 14-16, 2012.

[13] Miroslava Barúa, Homer Nazeran, Patricia Nava, Bill Diong, and Michael Goldman, (2005), "Classification of Impulse Oscillometric Patterns of Lung Function in Asthmatic Children using Artificial Neural Networks", Proceedings of the 2005 IEEE Engineering in Medicine and Biology 27th Annual Conference Shanghai, China, September 1-4, 2005, pp327-330.

[14] Yen, C.Y., (1999), "Rule selection in fuzzy expert system", Expert System with Applications, 16, pp 79-84.

[15] Chang-Shing Lee, Senior Member, IEEE, and Mei-Hui Wang, (2011), "A Fuzzy Expert System for Diabetes Decision Support Application", IEEE TRANSACTIONS ON SYSTEMS, MAN, AND CYBERNETICS—PART B: CYBERNETICS, VOL. 41, NO. 1, FEBRUARY 2011, pp139-153.

[16] Gopal Karemore, Jhinuk B. Mullick, R. Sujatha, Mads Nielsen, Santhosh C, (2010), "Classification of protein profiles using fuzzy clustering techniques:An application in early diagnosis of oral, cervical and ovarian Cancer", 32nd Annual International Conference of the IEEE EMBS Buenos Aires, Argentina, August 31 - September 4, 2010, pp6361-6364.

[17] J. E. Heiss, C. M. Held, P. A. Estévez, C. A. Perez, C. A. Holzmann, J. P. Pérez, "CLASSIFICATION OF SLEEP STAGES IN INFANTS: A NEURO FUZZY APPROACH".

[18] P. SANTOSH KUMAR PATRA, (2012), "Automatic Diagnosis of Diabetes by Expert System”, IJCSI International Journal of Computer Science Issues, Vol. 9, Issue 2, No 1, March 2012 ISSN (Online): 1694-0814, www.IJCSI.org, pp299-304.

[19] E.P.Ephzibah, Dr. V. Sundarapandian, (2012), "A NEURO FUZZY EXPERT SYSTEM FOR HEART DISEASE DIAGNOSIS “,Computer Science \& Engineering: An International Journal (CSEIJ), Vol.2, No.1, February 2012, pp17-23.

[20] M.Neshat1, M.Yaghobi, M.B.Naghibi, A.Esmaelzadeh, (2008), "Fuzzy Expert System Design for Diagnosis of liver disorders", proceeding IEEE 2008 International Symposium on Knowledge Acquisition and Modeling, pp252-256.

[21] Yuanyuan Chai, Limin Jia, and Zundong Zhang, (2009), "Mamdani Model based Adaptive Neural Fuzzy Inference System and its Application", International Journal of Information and Mathematical Sciences 5:1 2009, pp22-29.

[22] K. Balachandran, Dr. R.Anitha, (2010), "Supervisory Expert System Approach for Pre-Diagnosis of Lung Cancer", Published in International Journal of Advanced Engineering \& Applications, Jan. 2010, pp177-182. 